\title{
Section introduction \\ Introduction to the sessions on 'Debate: Extended follow-up of breast cancer patients in clinic wastes time for both patients and doctors'
}

\author{
Lesley Fallowfield ${ }^{1}$ and David George ${ }^{2}$ \\ ${ }^{1}$ Cancer Research UK, University of Sussex, Brighton \& Sussex Medical School, Falmer, Brighton, BN1 9QG, UK \\ 2Department of Surgery, Western Infirmary, University of Glasgow, Dumbarton Road, Glasgow, G11 6NT, UK
}

Corresponding author: Lesley Fallowfield I.j.fallowfield@sussex.ac.uk

Published: 18 December 2008

This article is online at http://breast-cancer-research.com/content/10/S4/S6 (c) 2008 BioMed Central Ltd

The motion for debate was, 'Extended follow-up of breast cancer patients in clinic wastes time for both patients and doctors'. Professor Mike Dixon spoke for the motion and Professor David Cameron spoke against it. Before the debate the delegates were asked to vote on the motion. This predebate vote revealed that 30 were in agreement with the motion, 36 disagreed and 13 were uncertain.

Speaking for the motion, Professor Dixon made the point that there is considerable discrepancy between the recommendations for follow-up from the UK and North America. He identified that there was no evidence base to guide the decision making, and hence follow-up had largely evolved on an ad hoc basis. Equally, there was no evidence that screening for distant metastases at follow-up clinics was of any value. Therefore, the main aims of follow-up would be to detect local or regional recurrence and perhaps to monitor for psychological morbidity. Potential methods for detecting such recurrences would include clinical examination at a follow-up clinic, mammography and the patient herself detecting an abnormality. He also highlighted that routine clinical examination at follow-up was of little value but accepted that mammographic surveillance was helpful. His recommendations were therefore that hospital follow-up is reasonable for the first 1 to 2 years after surgery, and thereafter surveillance should be by annual mammography and an annual questionnaire. This should be combined with discharge of the patient back to primary care.

Speaking against the motion, Professor Cameron also made the point that follow-up policy varied markedly between the UK and North America. He too agreed that there was no clinical trial evidence of the effect on outcome of a policy of regular follow-up versus no follow-up. He also emphasized strongly that detailed outcome information is mandatory for all patients enrolled in clinical trials, and that such information is best accrued through regular follow-up. The need to monitor
Breast Cancer Research 2008, 10(Suppl 4):S6 (doi:10.1186/bcr2166)

side effects of treatment, especially in relation to some of the more recently introduced therapies such as aromatase inhibitors (bone health) and trastuzumab (cardiac toxicity), was another important reason for maintaining longer follow-up. Additionally, patients find routine follow-up reassuring, and in one study $15 \%$ of patients requested review at the clinic during the year immediately after their discharge. Finally, Professor Cameron commented that discontinuing regular follow-up might not improve health economics, because the treatment of recurrence following delayed detection would be considerably more expensive than treatment of recurrence detected at an earlier stage. Similarly, treatment of osteoporosis-related fractures would be considerably more expensive than the medical management of early-stage osteoporosis detected through regular follow-up.

During the debate it became clear that both speakers felt that some form of follow-up was essential, and it was only on the question of methodology that they disagreed. Neither speaker defined 'extended', which clearly is of relevance.

Immediately after the debate the audience members were asked to vote on the motion again. On the second vote 32 agreed with the motion, 44 disagreed and three remained uncertain.

After the debate, the delegates were also asked to complete a questionnaire comprising five questions on various aspects of follow-up. The first question related to follow-up frequency. Only $11.4 \%$ supported a follow-up duration of 2 years or less. The majority (35.7\%) felt that follow-up should continue for as long as the patient requested. The second question probed the primary reasons for follow-up. Of the participants, $52.7 \%$ felt that every reason given was important - to check for recurrence, to ameliorate symptoms, clinical trial protocols and to reassure patients. The most important reason for two participants was to provide the treating physician with 
reassurance. The third question was, "Is follow-up beyond 2 years best done by primary care physicians?'; this was deemed inappropriate by $82 \%$ of respondents. The fourth question was, 'Does routine follow-up require senior medical staff?'; a total of $59.2 \%$ felt that this was not necessary. The final question was, 'Should follow-up be decided on an individual patient basis?'; a total of $92 \%$ felt that this was an appropriate approach.

From the evidence presented in the debate, the result of the votes and the results of the questionnaire, there is little doubt that there is general agreement that follow-up is essential and that this should be hospital based rather than through primary care. The major advantages of follow-up include the acquisition of outcome data, particularly in relation to clinical trials. Early detection of local or regional recurrence is important and is probably best monitored with regular mammography. The monitoring of side effects of treatment is important, particularly in relation to the early detection of preventable complications of such treatment. Total length of follow-up should certainly exceed 2 years, and ideally it should be patient centred and, where appropriate, individualized.

These views are at variance with both North American guidelines and the guidelines issued by the National Institute for Health and Clinical Excellence. They also raise the question of whether certain aspects of follow-up policy are suitable for study within the context of a prospective clinical trial. Furthermore, they raise questions regarding follow-up methodology in terms of nurse-led follow-up clinics, perhaps for those patients at low risk for recurrence, and more focused medical follow-up of those at high risk for recurrence.

Perhaps this debate will encourage us all to examine our own practice in the hope that new and more appropriate follow-up policies can be developed.

\section{Competing interests}

The authors declare that they have no competing interests.

\section{Acknowledgements}

This article has been published as part of Breast Cancer Research Volume 10 Supplement 4, 2008: Controversies in Breast Cancer 2008. The full contents of the supplement are available online at http://breast-cancer-research.com/supplements/10/S4 